\title{
Research on the Destruction System of Minitype Fuze Explosive Element
}

\author{
Qiang Xiao ${ }^{\text {a }}$, Bin Zhang \\ No. 63981 Unit of PLA, Wuhan 430311, China \\ a Xiaosongqiang8@163.com
}

Keywords: Programmable Logic controller (PLC), Fuse Explosive Element, Destruction System.

\begin{abstract}
Fuze detection is an important means for laboratory to accurately grasp the quality of fuze. After testing, a small amount of minitype fuze explosive elements will be left. Which need to be destroyed in time to eliminate potential safety hazard. Due to the shortcomings of traditional destruction equipment have problems, such as large volume, high site requirement and low automation level, in order to solve these problems, a destruction system for minitype fuze explosive element is developed based on PLC control. First, the general framework of the destruction system is given, and the parts of the system are designed in detail. Based on that, the operation mechanism and key technology implementation methods are determined. Finally, the feasibility and effectiveness of the design are verified by experiments. The study of the system not only can provide a theoretical basis for the destruction of fuze explosive element, but also has a certain reference value for the development of the destruction system.
\end{abstract}

\section{Introduction}

The test of ammunition components is a necessary method and means to monitor the quality of ammunition, and also a decision-making basis for the use, storage, ordering and technical treatment of ammunition. A certain amount of fuze initiating products remaining after the test are left. At present, the existing destroyed equipment mainly is used for medium-sized fuze exploding, which has features of big size, easy erection and low automation, time-consuming. How to destroy small explosive products efficiently and safely has always been the focus of our military equipment management department and the use department.

From the urgent needs of fuze exploding destruction, based on the actual situation, according to the actual structure and characteristics of loaded troops and Ammunition Fuze, a destruction system for Minitype Fuze Explosive Element is developed based on PLC control. A brief description of the system structure is given and, functional of the system is designed, on this basis, the operating mechanism and conduct the research on the key technology of system development is determined, finally, the destruction of the temperature is tested, and the test results are analyze[1].

\section{Overall Design of Destruction System}

\subsection{PLC Overview}

With the rapid development of control technology, as one of the advanced industrial computer control system, the PLC has been widely used in the military field, which brings good technical results and economic benefits to the military departments. Therefore, it is a practical and feasible method to study dynamical double environment force simulation system for Fuze [2].

PLC is oriented to the idea of digital logic operations to establish the control system, and the control system is considered as a "brain", each equipment of the control system is a module of the system, each module can relatively independent designed and develop to the maximum degree by itself in the field of advanced technology, and each module is provided with more network communication service interface layer for the operation of the PLC system, operation of the system is ensure efficient and accurate, and the three aspects of system function realization of the control algorithm program, operation management of equipment and underlying information interactive transmission is effectively separated, the operation, expansion of follow-up system can is improved 


\subsection{System Construction}

In the support of PLC, destruction system for Minitype Fuze Explosive Element is developed, from the demand and function of the detection system, the overall structure of system is divided into destruction device, slag removal device, PLC measurement and control system, temperature control device, counting device, man-machine interface etc six parts. the overall structure of system is shown in Fig 1.

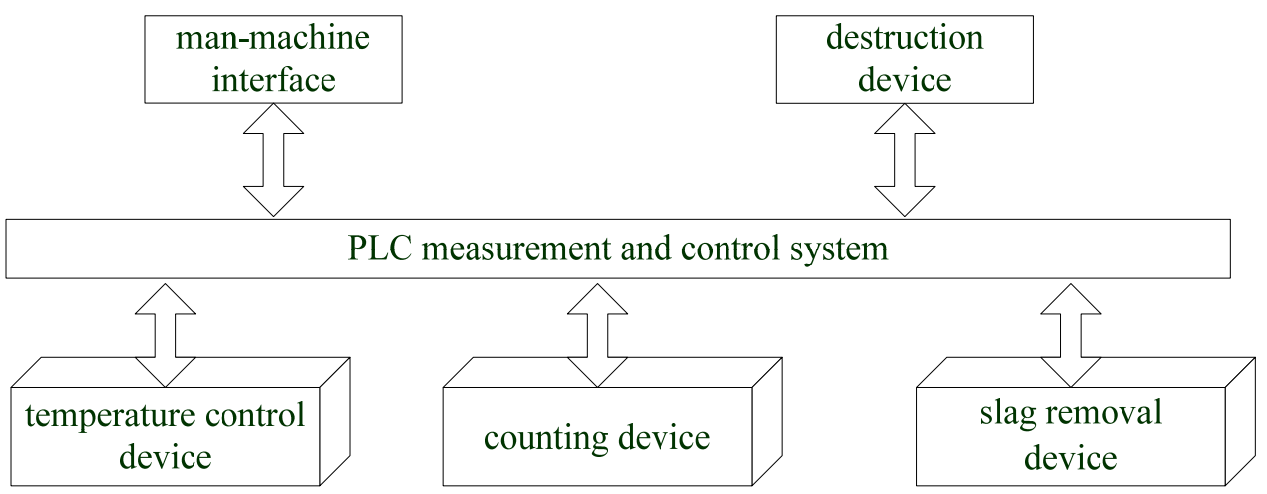

Fig. 1 The overall structure of destruction system

\section{Functional Design of Destruction System}

\subsection{Temperature Control Device}

This device is the key component of the destruction of fuze pyrotechnics, and the main function is to provide a suitable destruction environment by heating different fuze. Based on the PID algorithm, the closed loop control of heating temperature is carried out to ensure the control precision of the destruction temperature. The schematic diagram of temperature control device is shown in Fig 2

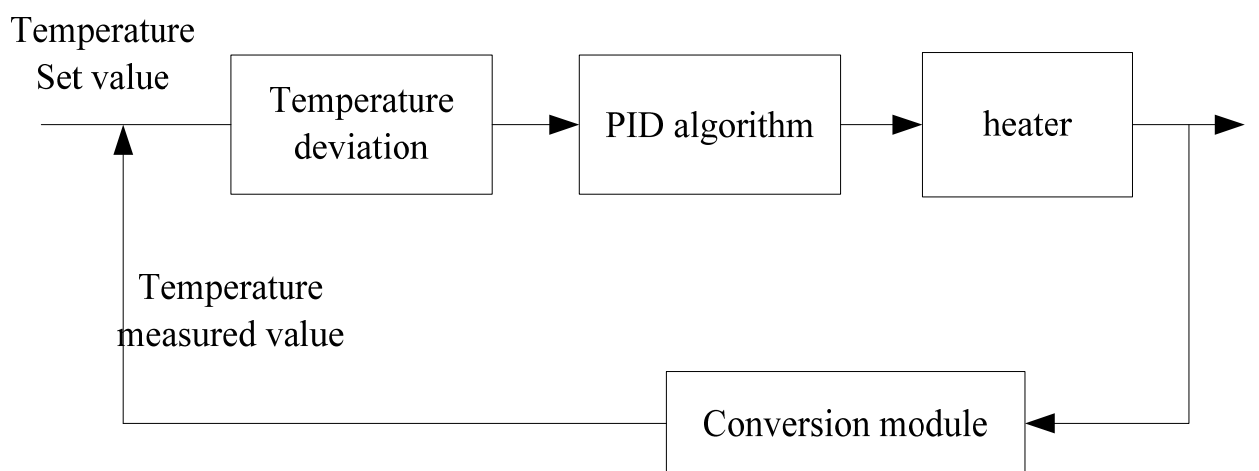

Fig. 2 The schematic diagram of temperature control device

The closed-loop dynamic negative feedback control mode is adopted to modify the rotation error automatically, and then high precision control are realized.

\subsection{Destruction Device}

The device is the destruction carrier of fuze explosive element, and the destruction temperature is rapidly increased by heating to realize the safe and efficient destruction of fuze explosive element.

\subsection{Counting Device}

The purpose of the device is used to determine the quality of the remnants of the fuze after the explosive detonation. Acquisition of fuze exploding blast sound signals is collected by the sound equipment, and quickly transferred to the control platform for voice signal analysis, recognition and counting. The fuze exploding destroyed and the destruction of the remaining residue is quality calculated, fuze exploding destruction state information is accurately obtained. The flow of counting device counting method is shown in Fig 3.

The main task of the target function system is used to simulating the environment of the target effect, and to finishing the test of the fire resistance performance of the fuze terminal trajectory. In the design, the system is mainly made of target shock device for simulating "target" impact fuze, pressure storage energy device for improving target impactor velocity, target guiding device which can be 
used to controling the effect of target direction of movement and electromagnetic unlocking device for controlling target impactor device attack time precisely, the system structure schematic diagram as shown in Fig 3.

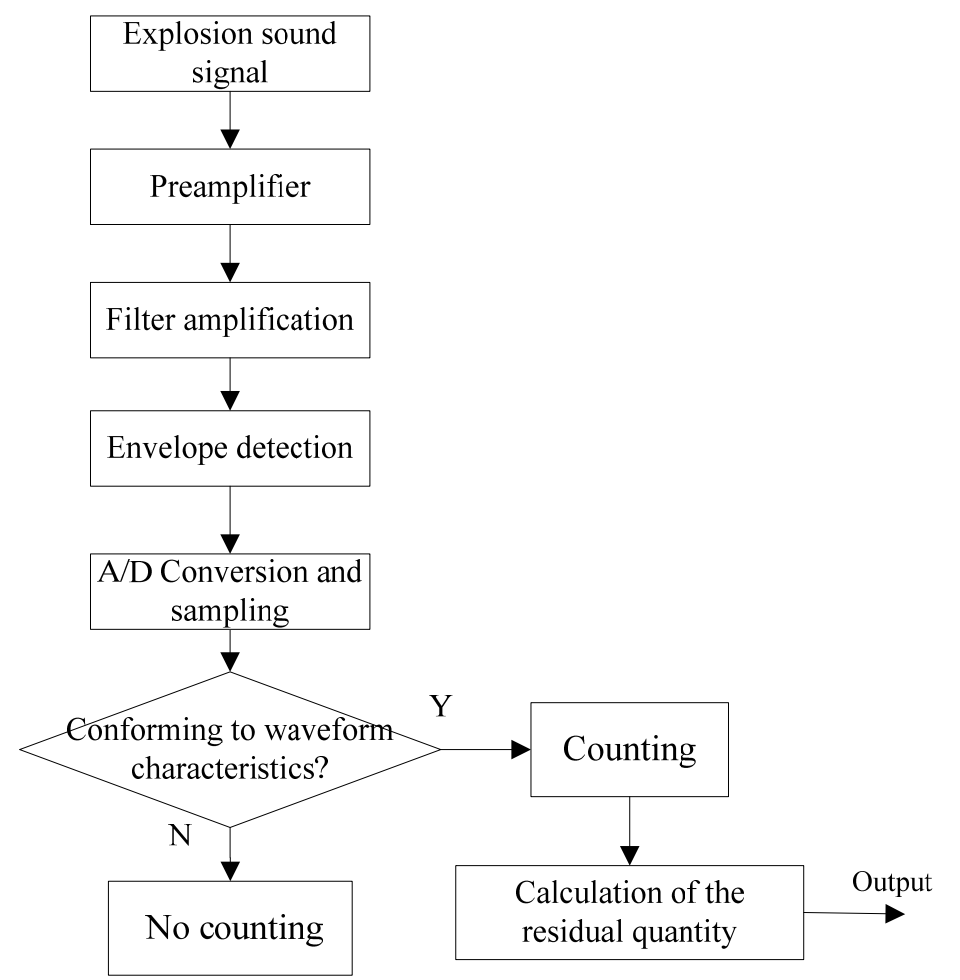

\subsection{Slag Removal Device}

Fig. 3 The structure schematic diagram of counting device

The device is mainly used for completing the angle and position recovery of the destruction device to clean up the residual material in the system in time. The electric flip design is adopted to control the pitch angle of the destruction device by receiving flip control instruction of the PLC control platform, and then achieve automatic and precise control of the destruction device, so as to ensure the efficient cleaning of the destruction device.

\subsection{Man-machine Interface}

It is interaction information window between human-computer that is mainly used to setting system initial state parameters, different types fuze explosive element test parameters, selecting operation mode, monitoring system running state and perform the system start, end, emergency stop and operation control in test process.

\subsection{PLC Measurement and Control System}

As the general prosecution of the system, the measurement and control platform is mainly responsible for the control and management of the whole system. It is the core part of the whole system. The performance of its measurement and control determines the automation and intelligence level of the fuze initiating product destruction. It is mainly used to complete the automatic measurement and control function of the system. Which realizes the precise control of slag removal system, temperature control system and counting device, and ensures the efficient operation of the destruction system.

\section{Test Verification}

In order to verify the scientificity of the system design, the PID temperature control test is carried out based on the PLC controller, and the comparison between the temperature and the actual PID control temperature is shown in Fig 4 and Fig 5. 


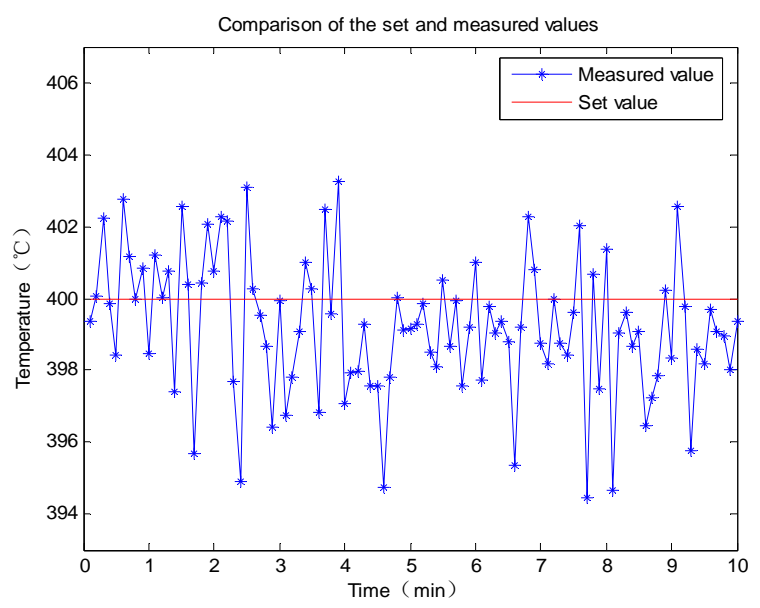

Fig. 4 the comparison diagram of temperature

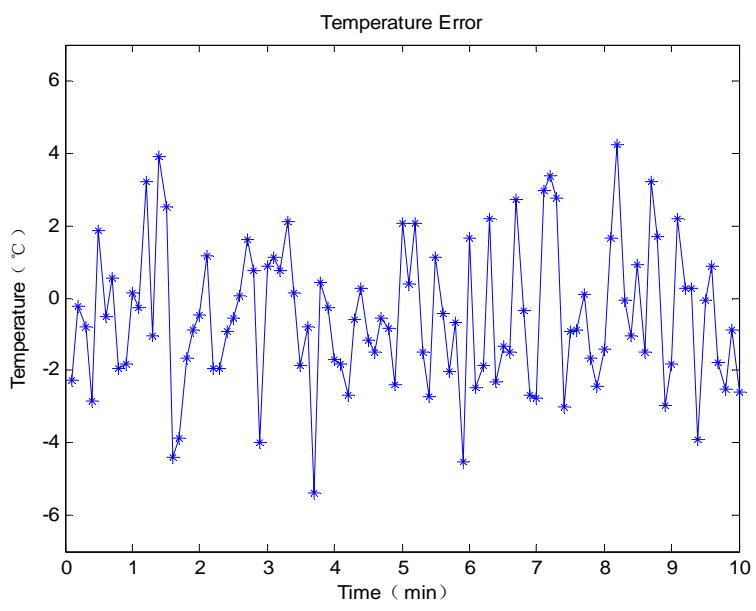

Fig. 5 the error of temperature

From Figure 5, Figure 6, the actual temperature set value is very consistent with the temperature measured value under PID control, and the error is less than $3 \%$, which indicates that the destruction temperature under PID control has high accuracy. Meanwhile, it is indicated that under the control of PID, the system can destroy the fuze initiator effectively.

\section{Conclusion}

Destruction system for minitype fuze explosive element is develop and designed by the PLC, through reasonable division of system and distribution function, and the operation mechanism of the system is analyzed in depth, which provides an effective and feasible method for destruction system for minitype fuze explosive element. Because this paper focuses on double environment simulation system design process, the specific system is only introduced briefly, but the design theory and method in the study is general. It has a certain reference military value to promote the rapid development of fuze explosive element destruction system. In the future research, the PLC measurement and control system specific programming development e n study will be carry out.

\section{References}

[1]. Liu Meng. Based on PLC and WinCC Fuze Automatic Assembly Aachine Control System Design [D]. Sichuan ordnance journal, Vol. 36(2015) No. 3, p.31-34.

[2]. .LV Pin. Application of PLC and Touch Panel Control System [J]. Automation Instrument. Vol. 31 (2010) No. 8, p.45-47 Vol. 31(2010) No. 8, p.45-47.

[3]. Wang Dong, Ma Qiang, et al. Anti-fragmentation enhanced blast furnace burning [fuze design [J].. Blasting equipment, Vol. 44(2015) No. 3,p. 60-63

[4]. Ren Junjie, Li Yongxia, Li Yuan, Li Hongxing. PLC based closed loop control system PID controller [J]. Manufacturing automation, Vol. 44(2009) No. 3.60-63.

[5]. Liu Pengan, Zhang Huaizhi et al. Theory and experiment of fuze burning model [J]. Journal of missile and arrows and guidance, Vol. 30(2010) No. 4. 92-95. 